\title{
Synthesis of (Polymer blend - MgO) Nanocomposites and Studying Electrical Properties for Piezoelectric Application
}

\author{
Majeed Ali Habeeb, Rehab Shather Abdul Hamza \\ Department of Physics, College of Education for Pure Sciences, University of Babylon, Iraq
}

\begin{tabular}{l} 
Article Info \\
\hline Article history: \\
Received Mei 28, 2018 \\
Revised Jul 31, 201 \\
Accepted Sep 11, 2018 \\
\hline
\end{tabular}

Keyword:

Electrical Properties

Nanocomposites

Piezoelectric Application

\begin{abstract}
Nanocomposites were prepared by the method of casting with different proportion of nano magnesium oxide $(0,1.5,3,4.5$ and 6$)$ wt $\%$.The structural and electrical properties of (PAA-CMC-MgO) nanocomposites were studied.The experimental results of Scanning electron microscopy shows the surface morphology of the (PAA-CMC-MgO) nanocomposites where many grouping or pieces randomly dole out on the top of surface, homogeneous and coherent. The D.C electrical conductivity for (PAA-CMC$\mathrm{MgO}$ ) nanocomposites increased with increasing of temperature and magnesium oxid nanoparticles concentration, while activation energy decreases with increasing of the magnesium oxid nanoparticles concentration. The A.C electrical properties show that the dielectric constant and dielectric loss of the nanocomposites decrease with increasing the frequency of applied electrical field and they increase with the increase of the concentration of the magnesium oxide nanoparticles. The A.C electrical conductivity increases with increasing the concentration of magnesium oxide nanoparticles and also increases with the increase frequency, as well as almost constant at high frequency. The outcomes of sensor application offered that the electrical resistance of (PAA-CMC-MgO) nanocomposite decreases with increases in pressure.
\end{abstract}

Copyright $@ 2018$ Institute of Advanced Engineering and Science. All rights reserved.

\section{Corresponding Author:}

Majeed Ali Habeeb,

Department of Physics, College of Education for Pure sciences,

University of Babylon, Hillah, Iraq.

Email: majeed-ali74@yahoo.com

\section{INTRODUCTION}

Nanotechnology involves is the science which includes the investigation and design of materials or devices at the atomic and molecular levels.nanotechnology deals with structure 100 nanometers or smaller, and involves developing materials or devices within that size [1], [2].

Many researchers have prepared polymer compositions and studied their applications in technology, medicine and the field of materials by using industrial polymers, but in this research, a new composite was prepared using biopolymer materials with nano-oxide and were studied their electrical properties and pizoelectric application.

Potential applications of nanotechnology: Ultra-light weight, high strength, barriers for thermal and optical application, faster, smaller and more efficient semiconductors and micro-processors, Nano-composite polymers and thin protective coatings for structural and electronic applications, low voltage and highbrightness displays, filters for cost-effective desalinization of water, miniaturized computers, non-volatile memory, antibacterial dressings and coatings, miniature thin film photovoltaic solar cells for cost effective power generation for applications ranging from laptop to automobiles, micro sensors and diagnostics for more effective treatment and highly effective drug compounds and perfectly targeted drug delivery [3], [4].

Sensor technology is one of the most common technologies for applications in the industry and medicine. It possible to be used in measure squeeze, temperature, quality, and the amount of energy, and to 
monitor health. Different kinds of sensors have been fabricated from polymer matrices for instance squeeze , thermal/infrared, vapor, humidity, gas, electrical, and temperature/thermal sensor. Piezoelectric sensors separate into two huge groups relying on their fundamental physical effects: (i) Sensors in the first group use a straight-line piezo effect. They are used for determining dynamic and quasistatic pressure, linear and vibrating accelerations, as well as parameters of sound and supersonic fields, etc. (ii) The second but no less general category of sensors concerns the so-called resonant piezo transducers, which use the return piezo effect. They are resonant sensors from piezoelectric resonators, and they can also make straight-line piezo effects. (These are resonant piezoelectric transformer sensors). Moreover, other physical effects can be used, e.g., thermosensitivity, acoustosensitivity, etc [5].

\section{EXPERIMENTAL PART}

Polymers nanocomposites have been prepared by casting technique by adding magnesium oxid (MgO) nanoparticles to $20 \%$ of poly acrylic acid (PAA) and $80 \%$ of carboxy methyl cellulose (CMC) with different weight percentages $(0,1.5,3,4.5$ and 6$) \mathrm{wt} \%$ in $30 \mathrm{ml}$ of water, by using magnetic stirrer. Sample dimensions include $1 \mathrm{~cm}$ for all samples and thickness $0.01 \mathrm{~cm} . \mathrm{MgO}$ nanoparticles it was obtained as powder from Nano shel USA company with grain size $20 \mathrm{~nm}$. The resistivity was recorded by temperature from (30 to 80) 0C by using keithley electrometer type (2400C) .The dielectric properties of nanocomposites were measured using LCR meter in the frequency(f) range $100 \mathrm{~Hz}-5 \mathrm{MHz}$ at room temperature. The piezoelectric application of nanocomposites investigated by measuring the resistance between two electrodes on the top and bottom of the sample for different pressures range (80-180) bar. The volume electrical conductivity $\sigma \mathrm{V}$ defined by [6]:

$$
\sigma_{\mathrm{v}}=\frac{1}{\rho_{\mathrm{V}}}=\frac{\mathrm{L}}{\mathrm{RA}}
$$

The activation energy was calculated using equation [7]:

$$
\sigma=\sigma_{0} \exp \left(-\frac{E_{\text {act }}}{\mathrm{K}_{\mathrm{B}} \mathrm{T}}\right)
$$

The dielectric constant $\left(\varepsilon^{\prime}\right)$ can be calculated by using the following equation [7]:

$$
\mathrm{C}_{\mathrm{P}}=\frac{\varepsilon^{\prime} \varepsilon_{\mathrm{o}} \mathrm{A}}{\mathrm{d}}
$$

whereas for dielectric loss $\left(\varepsilon^{\prime \prime}\right)$ can be calculated using equation [7]:

$$
\operatorname{Tan} \delta=\frac{\mathrm{I}_{\mathrm{p}}}{\mathrm{I}_{\mathrm{q}}}=\frac{\varepsilon^{\prime \prime}}{\varepsilon^{\prime}}
$$

The AC conductivity oac can be calculated by the following equation [8]:

$$
\sigma_{\mathrm{A} . \mathrm{C}}=\omega \varepsilon_{\mathrm{o}} \varepsilon^{\prime \prime}
$$

where,

$\mathrm{L}$ : Length polar

$\mathrm{R}$ : Resistance

A : Guard electrode effective area.

$\sigma$ : Electrical conductivity at $\mathrm{T}$ temperature

$\sigma_{0}$ : Electrical conductivity at absolute zero of temperature

$\mathrm{K}_{\mathrm{B}}$ : Boltzmann constant

$\mathrm{E}_{\mathrm{act}}$ : Activation energy.

$\mathrm{C}_{\mathrm{p}}$ : Capacitance

$\mathrm{d}:$ Sample thickness

$\varepsilon_{o}$ : Permittivity of free space or vacuum permittivity $\left(8.85^{*} 10^{-12} \mathrm{~F} / \mathrm{m}\right)$

A : Surface area of the sample

$\tan \delta$ : Dissipation factor

$\mathrm{I}_{\mathrm{p}}$ : Conduction current

$\mathrm{I}_{\mathrm{q}}$ : Capacitate current.

$\mathrm{w}:$ The angular frequency 


\section{RESULTS AND DISCUSSIONS}

\subsection{Scanning Electron Microscope (SEM) Measurements}

Figure 1 shows typical SEM images of the (PAA-CMC-MgO) nanocomposites films without and with different concentrations of magnesium oxide nanoparticles content. Image (A) in Figure1 found it more softer, homogenous and coherence. While, with increasing nanoparticles ratio step-by-step in polymer blends in the Figure1 (B,C,D,E) leads to changes in the surface morphology. The nanocomposites films show many spherical particles aggregates or chunks distributed and spread densely on the surface, this may be indicating the occurrence of a homogeneous growth mechanism [9]. The results indicate that the $\mathrm{MgO}$ nanoparticles tended to form aggregates and good dispersed at (PAA-CMC-MgO) nanocomposites films as shown in the Figure 1 (B,C,D and E). When adding $6 \mathrm{wt} \%$ of $\mathrm{MgO}$ nanoparticles to (PAA-CMC) composites, it form a continuous network inside the polymer[10], [11].
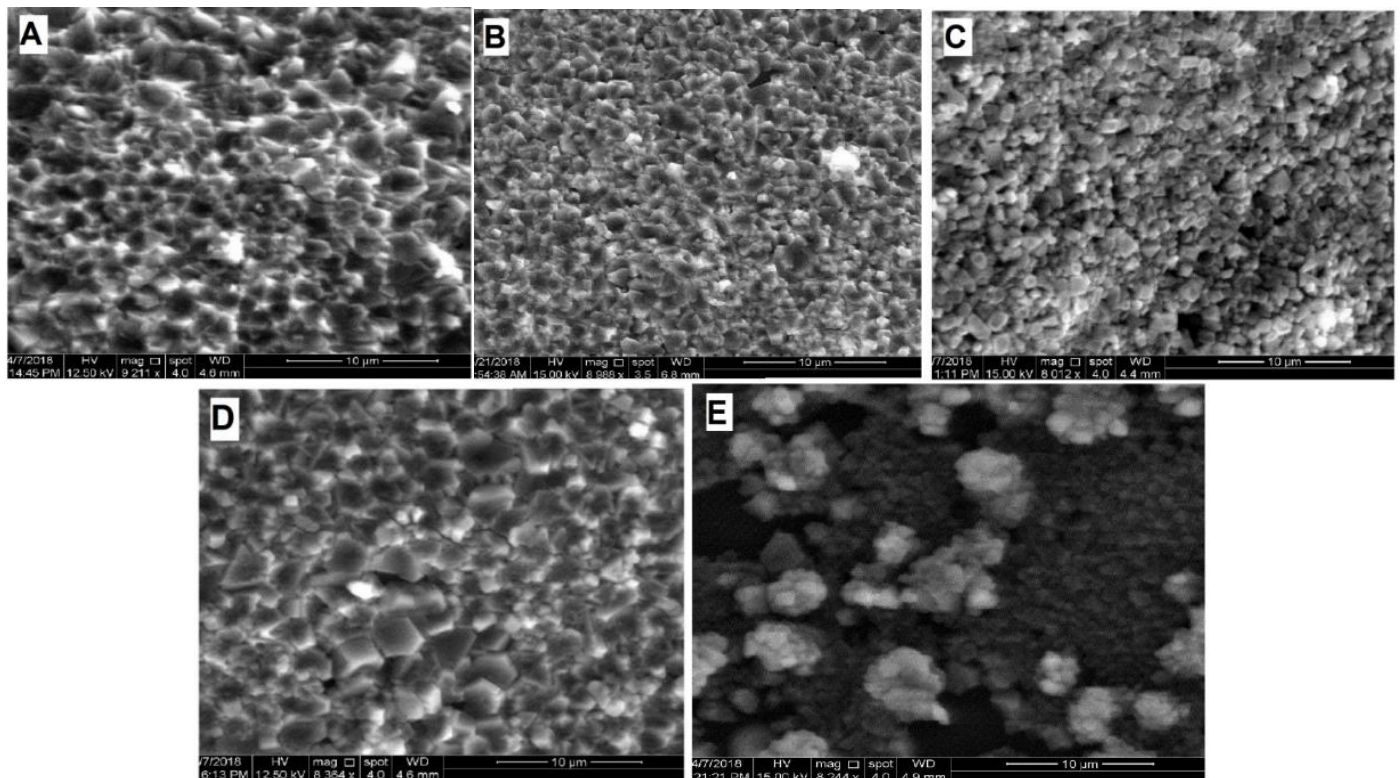

Figure 1. SEM images $(10 \mu \mathrm{m})$ for (PAA-CMC-MgO) nanocomposites: (A) for (PAA-CMC), (B) for $1.5 \mathrm{wt} \% \mathrm{MgO},(\mathrm{C})$ for $3 \mathrm{wt} \% \mathrm{MgO}$, (D) for $4.5 \mathrm{wt} \% \mathrm{MgO}$, (E) for $6 \mathrm{wt} \% \mathrm{MgO}$

\subsection{The D.C electrical properties(PAA-CMC-MgO) nanocomposites}

Figure 2 show the variation of D.C electrical conductivity of PAA and CMC as a function of magnesium oxide $(\mathrm{MgO})$ nanoparticles concentrations. From this figure, the electrical conductivity increases with the increase of the concentration of $(\mathrm{MgO})$ nanoparticles. The increase of conductivity can be explained as follows: at low concentration, the $\mathrm{MgO}$ nanoparticles are located in separated groups or cluster inside the polymer. when the concentration reaches to $6 \mathrm{wt}$ \% for (PAA-CMC-MgO) nanocomposites, the nanoparticles form a continuous network inside the polymer. This network has paths where charge carriers are allowed to pass through the paths that have low electrical resistance [12].

Figure 3 show that the electrical conductivity increases with increase of the temperature of (PAA$\mathrm{CMC}-\mathrm{MgO}$ ) nanocomposite this is means that resistance decrease with temperature increased. The explanation of this behavior is the polymeric chains and impurity ions act as traps to make charge carriers moving by hopping process this attributed to two main reasons the first one is electronic charge carriers and the second, mobility of these charges [12].

Figure 4 show the variation of Ln D.C electrical conductivity with inverted absolute temperature of nanocomposites. The activation energy was calculated by using (2) as shown in Figure 5. The activation energy has elevated values at low concentration because of the creation of topical energy levels in the forbidden energy gap which pretend to be as traps for charge carriers while at high concentration it has low values as aconsequence of the increase of local centers and the forming of a continuous network of $\mathrm{MgO}$ nanoparticles that expands paths inside the nanocomposite and allows charge carriers to pass through [13]. 


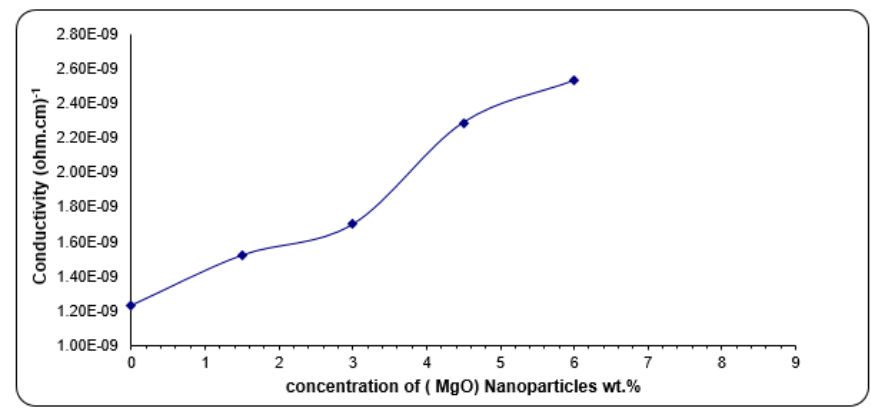

Figure 2.Variation of D.C electrical conductivity with $\mathrm{MgO}$ nanoparticles concentration for (PAA-CMC-MgO) nanocomposite.

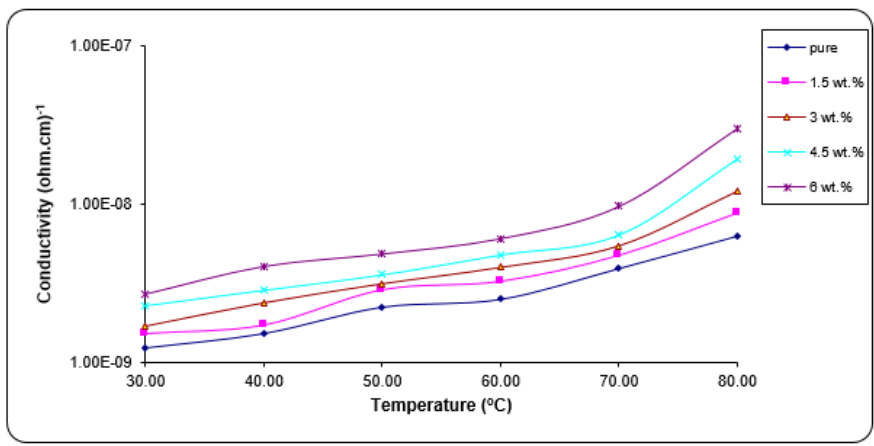

Figure 3.Variation of D.C electrical conductivity with temperature for (PAA-CMC-MgO) nanocompoites

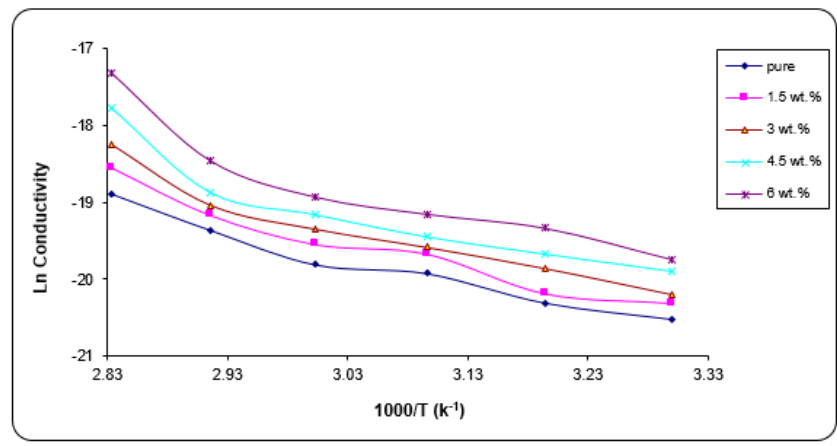

Figure 4.Variation of D.C electrical conductivity with inverse absolute temperature of (PAA-CMC-MgO) nanocomposites

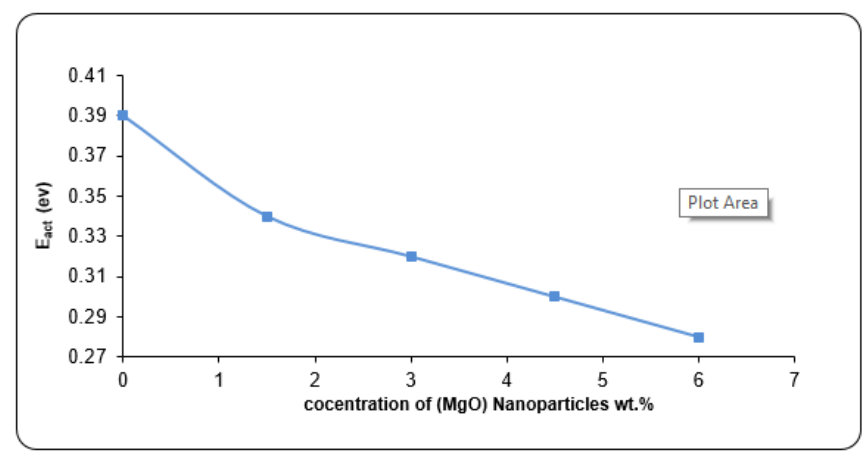

Figure 5. Variation activation energy for D.C electrical conductivity with nanoparticles $\mathrm{MgO}$ wt.\% concentration for (PAA-CMC-MgO) nanocomposites 


\subsection{The A.C electrical properties of (PAA-CMC-MgO) nanocomposites}

Figure 6 offers the variation of the dielectric constant of (PAA-CMC-MgO) nanocomposites with the frequency. From this figure the dielectric constant decrease when increasing the frequency This is refered to decreasing of space charge polarization with respect to the total polarization. The ionic polarization interacts somewhat to the variation in the field frequencies compared with electronic polarization; this is because the mass of ion is super than that of the electron. The electrons react even to the high frequencies of the field vibrations. The low mass of electron can make the electronic polarization was the only kind of polarization at higher frequencies, this leads to be the dielectric constant nearly constant for every samples at high frequencies. The dielectric constant is increased with the increase of the weight percentages of magnesium oxid nanoparticles which because of increase the charge carries numbers which is increase the electrical conductivity of polymer matrix [14], [15], as shown in Figure 7.

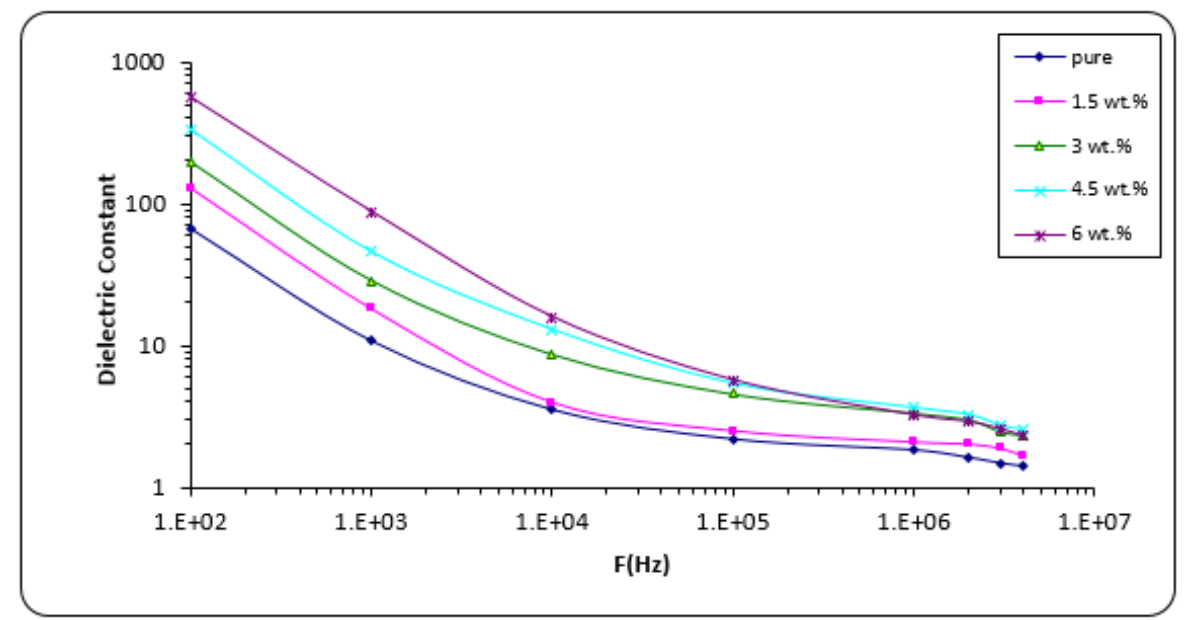

Figure 6. Variation of the dielectric constant of (PAA-CMC-MgO) nanocomposites with frequency

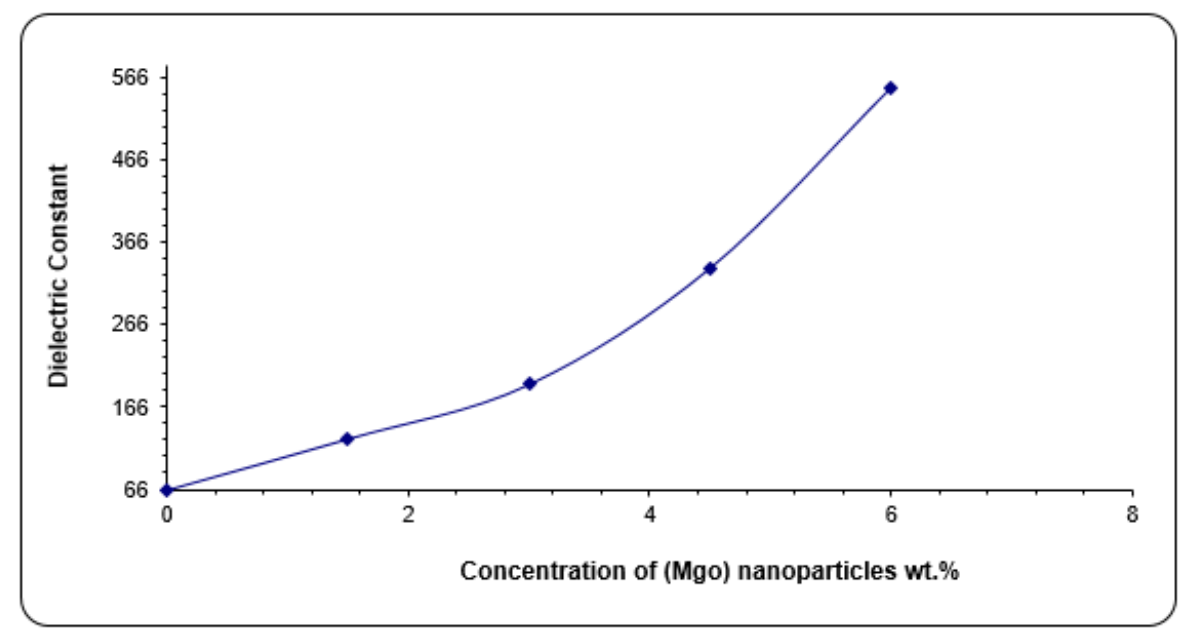

Figure 7. Variation of Dielectric constant with concentration of magnesium oxide nanoparticles wt.\% for (PAA-CMC-MgO) nanocomposites

Figure 8 displays the dielectric loss as a function of the frequency of (PAA-CMC-MgO) nanocomposites. From this figure the dielectric loss decreases with increase the frequency. This is because of the mobile charges within the polymer backbone. This is reffered to the decrease of the space charge polarization contribution when increasing the frequency. While the dielectric loss augments with the increasing of the concentration of $\mathrm{MgO}$ nanoparticles causes increase the electrons numbers of in nanocomposites [16], as shown in Figure 9. 


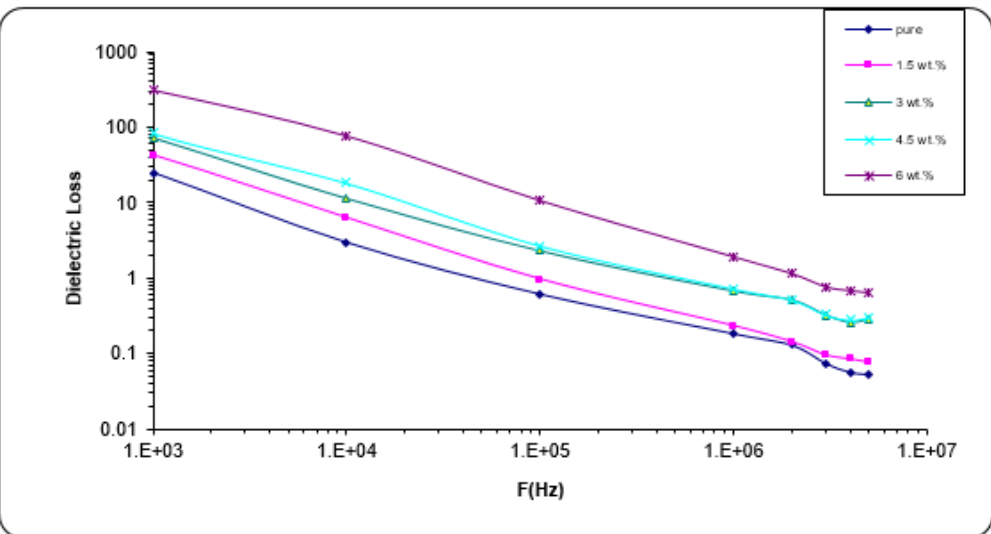

Figure 8. Variation of the dielectric loss with frequency for (PAA-CMC-MgO) nanocomposites

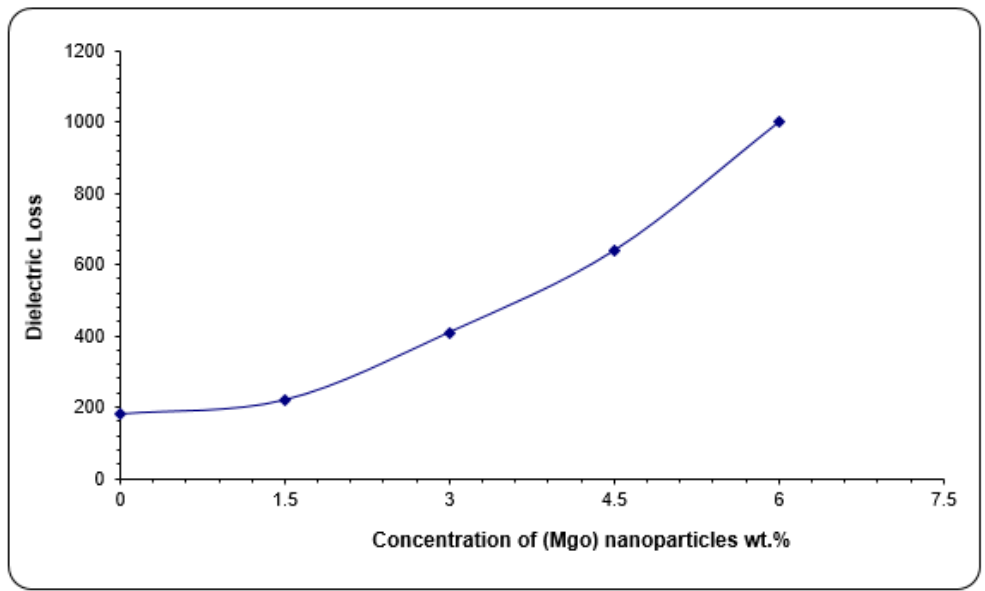

Figure 9. Variation of Dielectric loss with concentration of magnesium oxid nanoparticles wt.\% for (PAA-CMC-MgO) nanocomposites

Figure 10 show the variation of A.C electrical conductivity of nanocomposites as a function of frequency. As shown in figure the A.C electrical conductivity is increased with increase of the frequency, this is due to the space charge polarization. While the conductivity is increasing with the increase of the $\mathrm{MgO}$ nanoparticles concentration due to increase charge carrier density in polymer matrix [17], as shown in Figure 11.

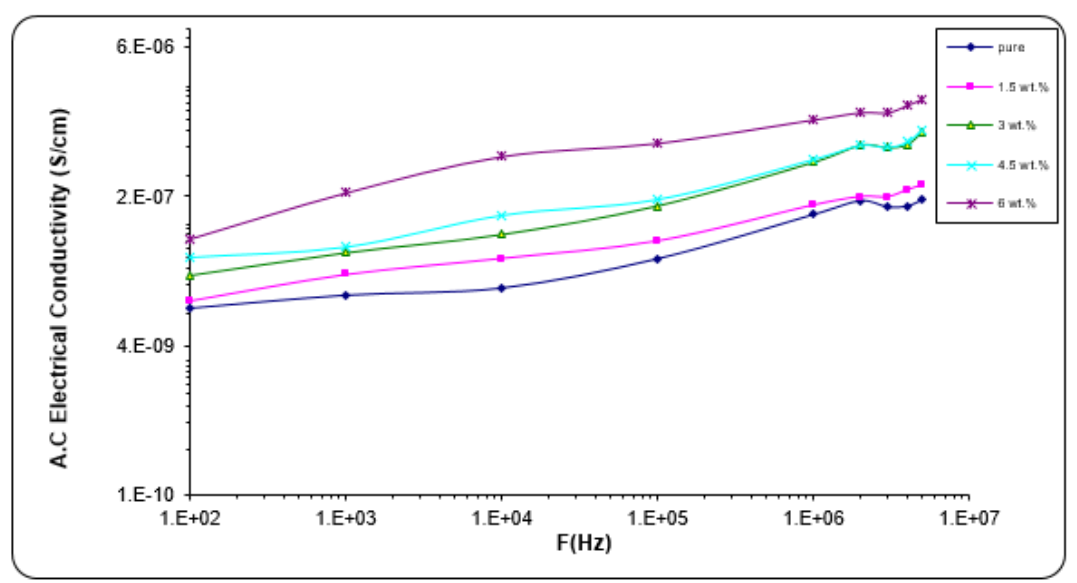

Figure 10. Variation of A.C electrical conductivity with frequency for (PAA-CMC-MgO) nanocomposites 


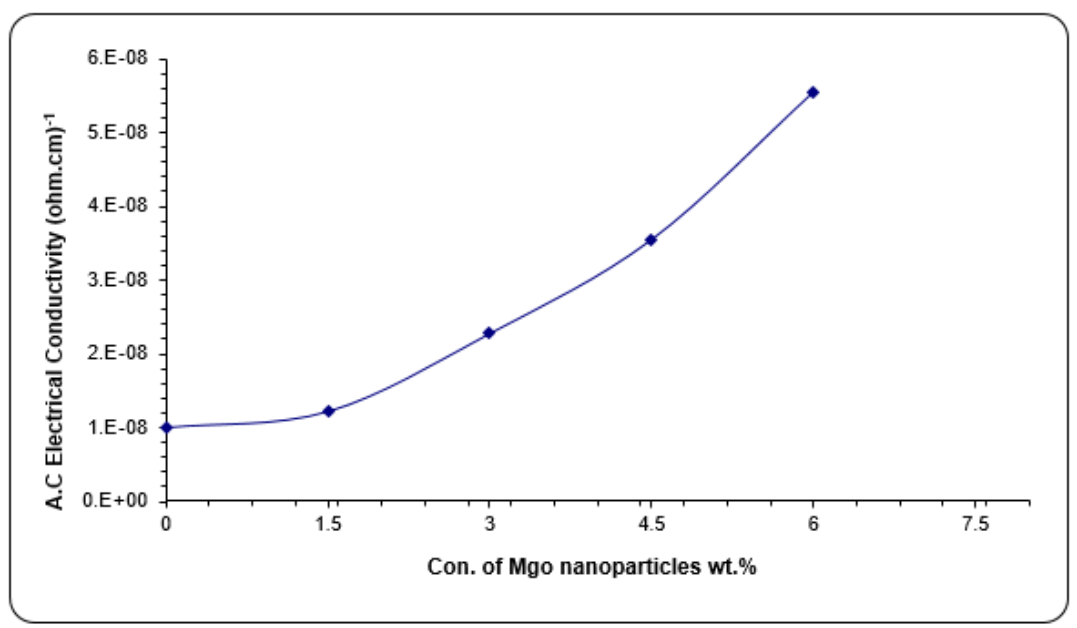

Figure 11. Variation of A.C electrical conductivity with magnesium oxid nanoparticles wt.\% concentration for (PAA-CMC-MgO) nanocomposites

\subsection{Piezoelectric Sensors Application}

Figure 12 displays the variation of electrical resistance for (PAA-CMC-MgO) nanocomposites with different pressure. The electrical resistance decreases with increase the pressure because the crystal contains of multiple interlocking domains consisting of positive and negative charges. These domains are symmetrical within the crystal. When a stress is applied to the crystal this symmetry is broken so the domains will reorder themselves in order to restore the symmetry, through this process, will generate a current and the resistance will be decreased [18].

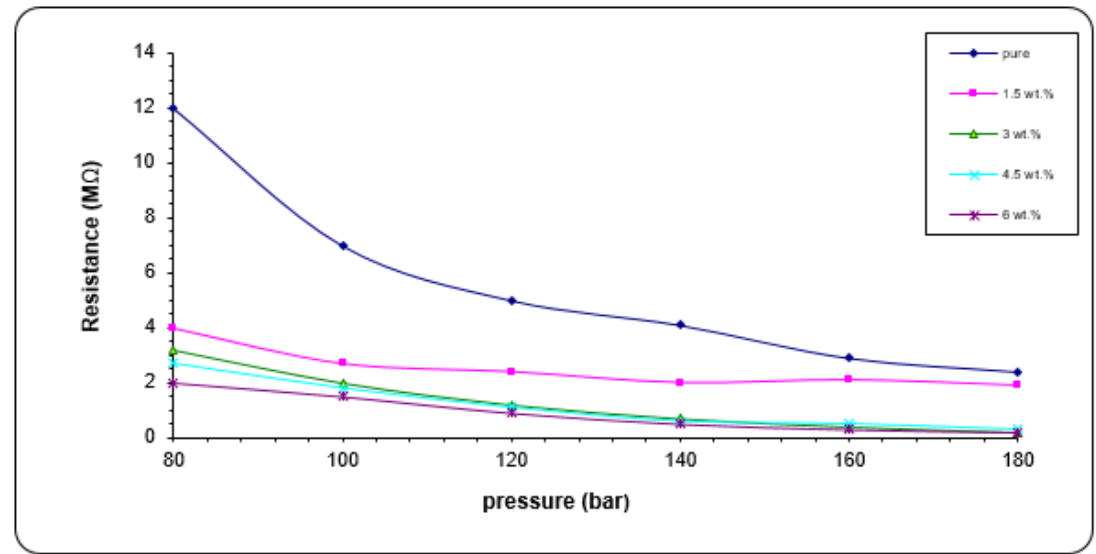

Figure 12. Variation of electrical resistance with pressure

\section{CONCLUSION}

Scanning electron microscopy (SEM) shows the surface morphology of the (PAA-CMC-MgO) nanocomposites films many grouping or pieces randomly dole out on the top of surface, homogeneous and coherent. D.C electrical conductivity for (PAA-CMC-MgO) nanocomposites increased with increasing of temperature and magnesium oxid nanoparticles wt.\% concentration. Activation energy of (PAA-CMC-MgO) nanocomposites decreases with increasing of the magnesium oxid nanoparticles wt.\% concentration. The dielectric constant, dielectric loss and A.C electrical conductivity of (PAA-CMC-MgO) nanocomposites increase with increasing of the $\mathrm{MgO}$ nanoparticles concentrations. The dielectric constant and dielectric loss of (PAA-CMC-MgO) nanocomposites decrease with increase the frequency, while A.C electrical conductivity increases with increase the frequency. The (PAA- $\mathrm{CMC}-\mathrm{MgO}$ ) nanocomposites have high sensitivity for pressure. 


\section{REFERENCES}

[1] J.C. Miller, R. Serrato, J.M. Represas-Cardenas and G. Kundahl,"the handbook of nanotechnology," Published by John Wiley and Sons, Inc., Hoboken, New Jersey, part 1, USA, 2005.

[2] M.A. Habeeb and L.A. Hamza, "Structural, Optical and D.C Electrical Properties of (PVA-PVP-Y2O3) Films and Their Application for Humidity Sensor," Journal of Advanced Physics, vol. 6, pp. 1-9, 2017.

[3] K. A. Shenoy, "Effects of Multi-Wall Carbon Nanotubes on The Mechanical Properties Of Polymeric Nanocomposites," M.Sc. Thesis, Department of Mechanical Engineering and the faculty of the Graduate School of Wichita State University, 2008.

[4] M. A. Habeeb and W. K. Kadhim, "Study the optical properties of (PVA-PVAC-Ti) Nanocomposites," Journal of Engineering and Applied Sciences, vol. 9(4), pp. 109-113, 2014.

[5] A. Hashim, M. A. Habeeb, A. Khalaf and A. Hadi, "Synthesis of Novel (Polymer Blend-Titanium Carbide) Nanocomposites and Studying their Characterizations for Piezoelectric Applications," Journal of University of Babylon, Pure and Applied Sciences, vol. 26(6), 2018.

[6] I.R. Agool, K. J. Kadhim and A. Hashim, "Synthesis of (PVA-PEG-PVP-MgO) nanobiomaterials and their application," Advances in Environmental Biology, vol. 9, pp. 101-106, 2015.

[7] M. A. Habeeb and W. K. Kadhim, "Study the Electrical properties of (PVA-PVAC-Ti) Nanocomposites," Australian Journal of Basic and Applied Sciences, vol. 8(10), pp.316-322, 2014.

[8] H. Frohlich, "Theory of Dielectric," 2nd ed., Oxfored University press, 1988.

[9] S. Ravichandran, "Synthesis and enhanced mechanical properties of nano Zinc Oxide in Polyvinyl alcohol and polyvinyl pyrollidone composite film," Int. J. Nano Dimens, vol. 4(2), pp. 153-159, 2013.

[10] E. Sheha, H. Khoder, T.S. Shanap, M.G. El-Shaarawy and M.K. El Mansy, "Structure, dielectric and optical properties of p-type (PVA/CuI) nanocomposite polymer electrolyte for photovoltaic cells," University of Benha, Egypt, vol. 123(13), pp. 1161-1166, 2012.

[11] N. K. Abbas, M. A. Habeeb and A. J. K. Algidsawi, "Preparation of Chloro Penta Amine Cobalt (III) Chloride and Study of Its Influence on the Structural and Some Optical Properties of Polyvinyl Acetate," International Journal of Polymer Science, vol. 2015, pp. 10, 2015.

[12] J. Gurland, "Electrical resistivity," J. Polym. Plast. Technol, Eng, vol. 19(1), pp. 21-51, 1982.

[13] M.A. Habeeb, A. Hashim, A. Khalaf and A. Hadi, "Fabrication of (PVA-PAA) Blend-Extracts of Plants BioComposites and Studying Their Structural, Electrical and Optical Properties for Humidity Sensors Applications," Sensor Letters, vol.15(7), pp. 589-596, 2017.

[14] C. Chiteme, S. Lowther and S. Harrison, "Science of Polymer," vol. 43, pp. 3273-3287, 2005.

[15] M. A. Habeeb, "Dielectric and optical properties of (PVAc-PEG-Ber) biocomposites", Journal of Engineering and Applied Sciences, vol.9, No.4, pp.102-108, 2014.

[16] A. Hashim, M. A. Habeeb, A. Hadi and W. Hadi, "Fabrication of Novel (PVA-PEG-CMC-Fe 3 O 4 ) Magnetic Nanocomposites for Piezoelectric Applications," Sensor Letters, vol.15(12), pp. 998-1002, 2017.

[17] G. Shui, J. Hu, M. Qiu, M. Wei, D. Xiao and J. Chinese chem, "Study of Dielectric Properties For (Calcium Oxidepoly-vinyl alcohol) Composites," letters, vol. 15(12), pp. 1501-1504, 2004.

[18] M. A. Habeeb, A. Hashim and A. Hadi, "Fabrication of New Nanocomposites: CMC-PAA-PbO2 Nanoparticles for Piezoelectric Sensors and Gamma Radiation Shielding Applications," Sensor Letters, vol. 15(9), pp. 1-6, 2017. 